\title{
IFprofs: le réseau des professionnels de l'éducation en français
}

\section{Marion Dufour}

\section{(2) OpenEdition \\ Journals}

Édition électronique

URL : http://journals.openedition.org/esp/3467

DOI : $10.4000 /$ esp.3467

ISSN : 2532-0319

Éditeur

Centre d'Information sur l'Éducation Bilingue et Plurilingue

\section{Édition imprimée}

Date de publication : 1 décembre 2018

Pagination : 108-109

ISSN : 1127-266X

\section{Référence électronique}




\title{
IFPROFS: LE RÉSEAU DES PROFESSIONNELS DE L'ÉDUCATION EN FRANÇAIS
}

\author{
Marion Dufour (1) \\ Dal 2015 l'Institut français Paris si sforza di soiluppare una rete sociale mondiale di professiomisti del mondo dell'istru- \\ zione in francese, che raggruppa soprattutto insegnanti, futuri insegnanti, formatori, ricercatori, consulenti pedagogici, responsa- \\ bili d'istituto e delle mediateche. \\ Parole chiave: IFprofs, rete professionale, professionisti dell'istruzione, mutualizzazione delle risorse, partenariato, \\ Istituto francese, formazione \\ Since 2015, the French Institute in Paris has been developing a worldwide social network for the professionals of education in \\ French, bringing together teachers, future teachers, trainers, researchers, pedagogical consultants and those in charge of cultural \\ institutions and media libraries.
}

Key-words: IFprofs, worldwide social network, education professionals, pooling resources, French Institute, training

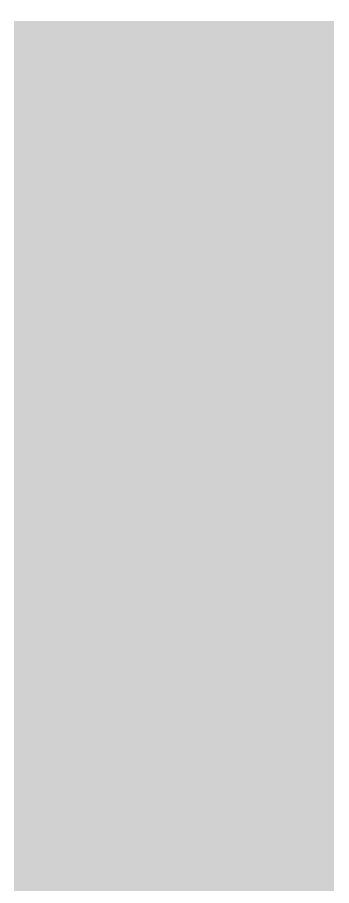
uis 2015, l'Institut français Paris s'emploie à développer un réseau ocial mondial des professionnels de l'éducation en français, regroupant notamment enseignants, futurs enseignants, formateurs, chercheurs, conseillers pédagogiques, responsables d'établissement et médiathécaires.

À l'origine de ce projet de plateforme collaborative, baptisée «IFprofs» (Institut Français Professionnels), se trouve la volonté, d'une part, de promouvoir la langue française comme une des grandes langues relais de la mondialisation et, d'autre part, d'offrir aux professionnels de l'éducation en français un espace unique, où échanger leurs informations et mutualiser leurs ressources pédagogiques et méthodologiques, plutôt que les voir se disperser sur l'ensemble des sites collaboratifs de la toile.

En facilitant la mise en relation des professionnels de l'éducation appartenant à différentes régions du monde, divers secteurs d'activités, différents degrés du système éducatif, et chargés de l'enseignement de différentes disciplines en français à des apprenants de différents niveaux, IFprofs se veut transversal et souhaite répondre aux besoins de mobilité, d'échanges et de formation continue de ces professionnels.

Plus concrètement, IFprofs est structuré en sites-pays qui servent d'interfaces entre les membres du réseau. Tous les professionnels inscrits, quel que soit leur site-pays, peuvent ainsi entrer en relation et communiquer avec les membres de leur choix par l'intermédiaire d'un forum. Ils peuvent aussi former des groupes de travail ou rejoindre des groupes existants: intercompréhension en langues romanes, Français langue seconde, 


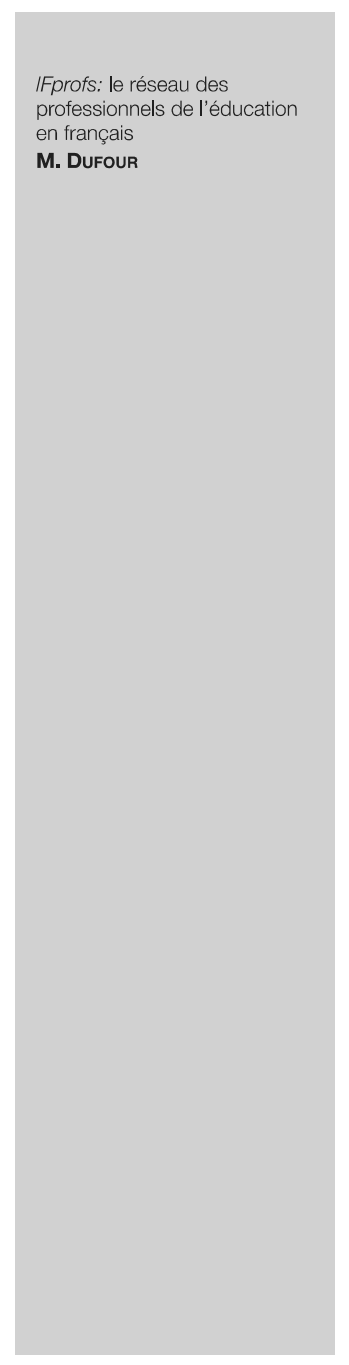

sciences et technologies en français, enseignants du supérieur, assistants de français du Royaume-Uni, etc.

Chaque site-pays offre la possibilité à ses membres de publier sur la plateforme des ressources de leur cru dans trois rubriques distinctes: ressources pédagogiques, ressources méthodologiques, et ressources brutes, cette dernière rubrique regroupant des textes non didactisés. Les auteurs de ressources ont le choix de les mettre à la disposition de tout le réseau IFprofs ou bien d'un ou plusieurs groupes de destinataires. De la même manière, ils peuvent faire circuler sur le réseau des informations comme des offres d'emplois, des appels à communication, etc., et des évènements relatifs à l'éducation en français: journée de la francophonie, lancement d'un MOOC (Massive Open Online Courses) c'est-à-dire d'une formation gratuite accessible à tous sur internet, etc.

Deux cent cinquante-sept animateurs, recrutés sur place par les attachés de coopération, sont actuellement chargés d'insuffler aux membres de leur site-pays une dynamique de communication et de publication, et de développer des partenariats avec des organismes numériques et institutions locales partageant les mêmes préoccupations de promotion de l'éducation en français: des bibliothèques numériques, des applications ludoéducative d'apprentissage des langues, des journaux éducatifs, des fondations menant des opérations pédagogiques en français, des centres et programmes de formation en langues, des pôles de ressources et formation spécialisés dans l'éducation plurilingue, des projets artistiques autour de la langue française et de la francophonie, des éditeurs spécialistes des langues, etc. La visibilité offerte sur la plateforme IFprofs à ces partenaires leur permet de faire connaitre leur action et d'étendre leur réseau. De son côté, les partenaires s'engagent à participer à la vie du réseau IFprofs sous la forme de publications et d'initiatives d'animation/présentation, en proposant par exemple un jeu concours ou en participant à un des webinaires organisés par Coline Durand (2), chargée de mission numérique éducatif chef de projet IFprofs.

Après plus de trois ans d'effort, IFprofs compte aujourd'hui plus de 21,000 membres et se déploie actuellement dans 67 pays, sur les cinq continents. Le Plan stratégique pour la langue française et le plurilinguisme du Président français Emmanuel Macron, rendu public le 20 mars 2018, prévoit un déploiement massif d'IFprofs au cours des trois prochaines années, avec une cible de 150 pays et 75,000 adhérents en 2021.

(1) Marion Dufour est l'animatrice du site IFprofs du Royaume-Uni (IFprofsUK)

(2) Coline Durand s'occupe également des partenariats et de la préparation de l'ouverture des sites-pays (coline.durand@institutfrancais.com)

Pour rejoindre le réseau cliquer sur: www.ifprofs.org 